\title{
Influence of the Angle between Ridge and Wind Direction on Wind Speed Distribution in the Wind Farm under Complex Topography
}

\author{
Xiangdong Zhu ${ }^{1, a}$, Gang Fan ${ }^{1, b}$,DezhengNing ${ }^{2}$, Huaikong Zhang ${ }^{2}$, Shouyuan \\ $\mathrm{Fan}^{2}$ \\ ${ }^{1}$ Faculty of Metallurgical and Energy Engineering, Kunming University of Science and Technology, \\ Kunming 650093, China \\ ${ }^{2}$ Yunnan Electric Power Design Institute, Kunming 650051, China \\ amcloud2028@foxmail.com, ${ }^{\mathrm{b}} \mathrm{kgfangang2008@sina.com}$
}

Keywords:Complex terrain, Wind farm, wind speed distribution, shunt phenomenon, Computational fluid dynamics.

\begin{abstract}
There are various inclined angles between mountain ridge and wind direction in the wind farm under the condition of complex topography. It is next to impossible to simulate the shunt phenomenon in wind acceleration process use commercial software, especially the mutations of wind speed distribution in the situation of wind direction parallels the mountain ridge. In this article, the wind speed distribution was studied by comparative test result when there is a smaller angle of wind direction and mountain ridge. The result of this study can provide a scientific and detailed reference for macro and micro sitting for large wind farms.
\end{abstract}

\section{Introduction}

Recently, great efforts have been devoted to the study of micro sitting for wind farms. Various directions of mountain ridge is a common phenomenon in complex terrain. In addition, the acceleration effect of mountain ridge to the wind varies significantly with the inclined angles between them. Related research reports are few due to the complexity. As to the wind energy resource sustainable development, wind farm siting is trend to the complex terrain with poor construction condition as well as high turbulent scale. So the wind resource assessment software used linear model are not suitable to analyze the wind resources under the condition of complex topography. Many engineers turned to the wind resource assessment using the method of computational fluid dynamics over the past few years. ReNE CATTIN et al. ${ }^{[1]}$ carried out the simulation analysis of wind resource in the Alps region. They also contrast and analyze the simulation result and meteorological station data. The research result shows that the CFD software is more accurate than the linear model method software, especially in the complex terrain ${ }^{[2 \sim 3]}$.

However, the CFD model may produce large errors in some complex terrains, such as the ridge direction or roughness or windward slope degree change dramatically. This article contrasted and analyzed the CFD simulation results and the actual wind data, summarized the wind distribution regularities while the ridge direction changed.

\section{CFD Model and Experiments}

Computational fluid dynamics (CFD) has been used extensively in many fields of industry along with the development of the computer science and fluid mechanics theory recently. The fundamental basis of almost all CFD problems are the Navier-Stokes equations, which define any single-phase (gas or liquid, but not both) fluid flow. During preprocessing, the geometry (physical bounds) of the problem should be defined, the volume occupied by the fluid is divided into discrete cells (the mesh), the physical modeling is defined - for example, the equations of motion + enthalpy + radiation + species conservation ${ }^{[4 \sim 6]}$. And boundary conditions are defined, this involves specifying the fluid behaviour and properties at the boundaries of the problem. Then, the simulation is started and the 
equations are solved iteratively as a steady-state or transient. Finally a postprocessor is used for the analysis and visualization of the resulting solution ${ }^{[7,8]}$.

In the experiment, three wind turbines (named 30, 31 and 32, respectively) in a typical wind farm were studied as shown in Fig.1. The effect of different angles between ridge and wind direction on the wind velocity variation was studied with CFD method.

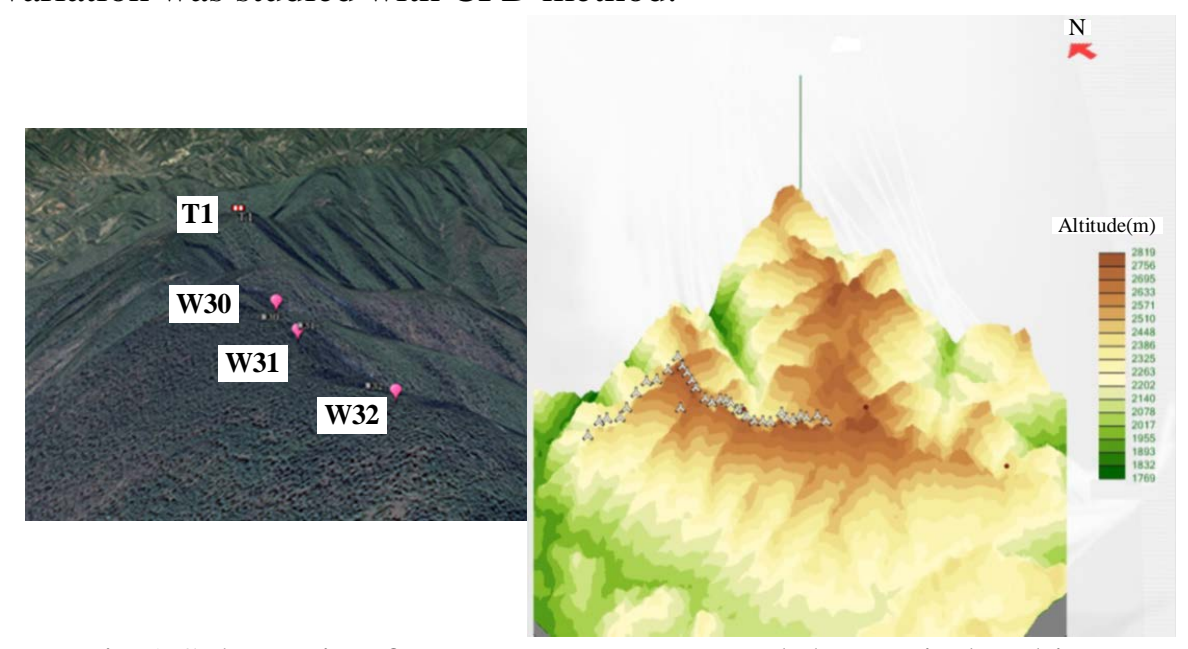

Fig.1 Schematic of anemometer tower and three wind turbines

As shown in Fig.1, the three wind turbines are located on a ridge and vary within $20 \mathrm{~m}$ in altitude. The azimuth angle of the ridge is 315degree (clockwise). The distance between the anemometer tower (named T1) and NO.30 wind turbine is $2.3 \mathrm{~km}$. And Altitude difference of them with $30 \mathrm{~m}$. The anemometer tower and the three wind turbines are under the same climatic condition, no shelter around. In the experiment, the measured data from T1 are used to calculate the wind speed and direction distribution in various sectors of $\mathrm{T} 1$ and the three wind turbines with commercial CFD software. The fixed wind sector step is 22.5 degree.

\section{Results and Discussion}

\subsection{Simulation Analysis}

Considering that the effect of incoming wind flow speed on observation point wind speed vary with the wind direction, the study of acceleration factor is implemented first. Simultaneously, in order to simplify the model, the influence of the landform and physiognomy is considered only, and the influence of the turbulence intensity change caused by different wind speed or thermal stability conditions is ignored.

Acceleration Factor. The compared curves of the acceleration factor in various sectors are shown in Fig.2.

It can be seen from Fig.2 that the acceleration factor has significant correlation with the angle between ridge and wind direction, the smaller the angle is, the smaller the acceleration factor is. In addition, the 90 degree inclined angle may be the optimum conditions.

Relationship between Wind Direction and Wind Speed. Fig.3 shows the normalizedwind speed distribution in various sectors under the condition of incoming flow speed is $10 \mathrm{~m} / \mathrm{s}$. Obviously, the variation tendency is different with the acceleration factor. It is seen from Fig. 3 that the normalized wind speed is relatively small while the included angle is less than 45degree (azimuth angle is 135 or 315 degree, marked in Fig.2). Even more interesting is that the least value is not present to the included angle of 0 but nearly 22.5 degree.

Acceleration Effect at Different Wind Speed. Contrast curves of the research three wind turbines under different incoming flow wind speed are shown in Fig.4. It can be seen from Fig. 4 that the acceleration effect under different incoming flow wind speed shows a good concordance with the acceleration factor, and its significant variation period is nearly 180 degree. 


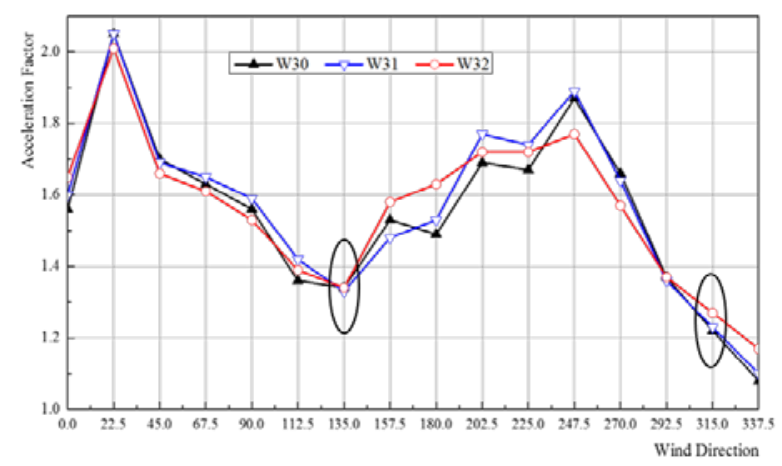

Fig.2 Compared curves of the acceleration factor in various sectors

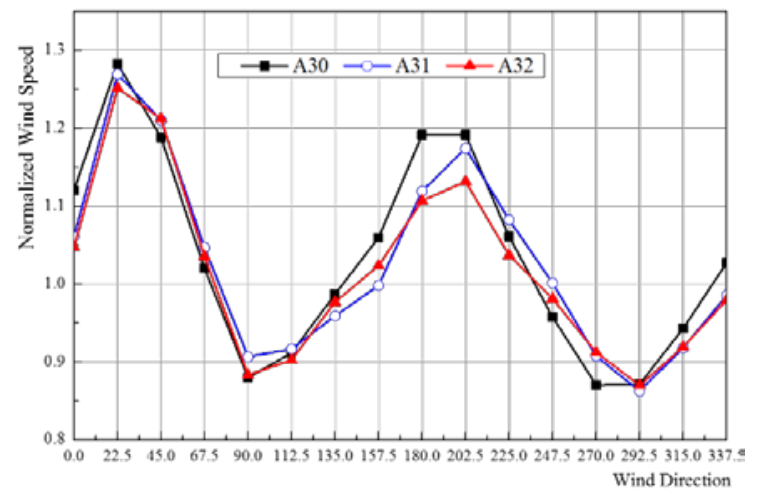

Fig.3 Normalized wind speed distribution in various sectors
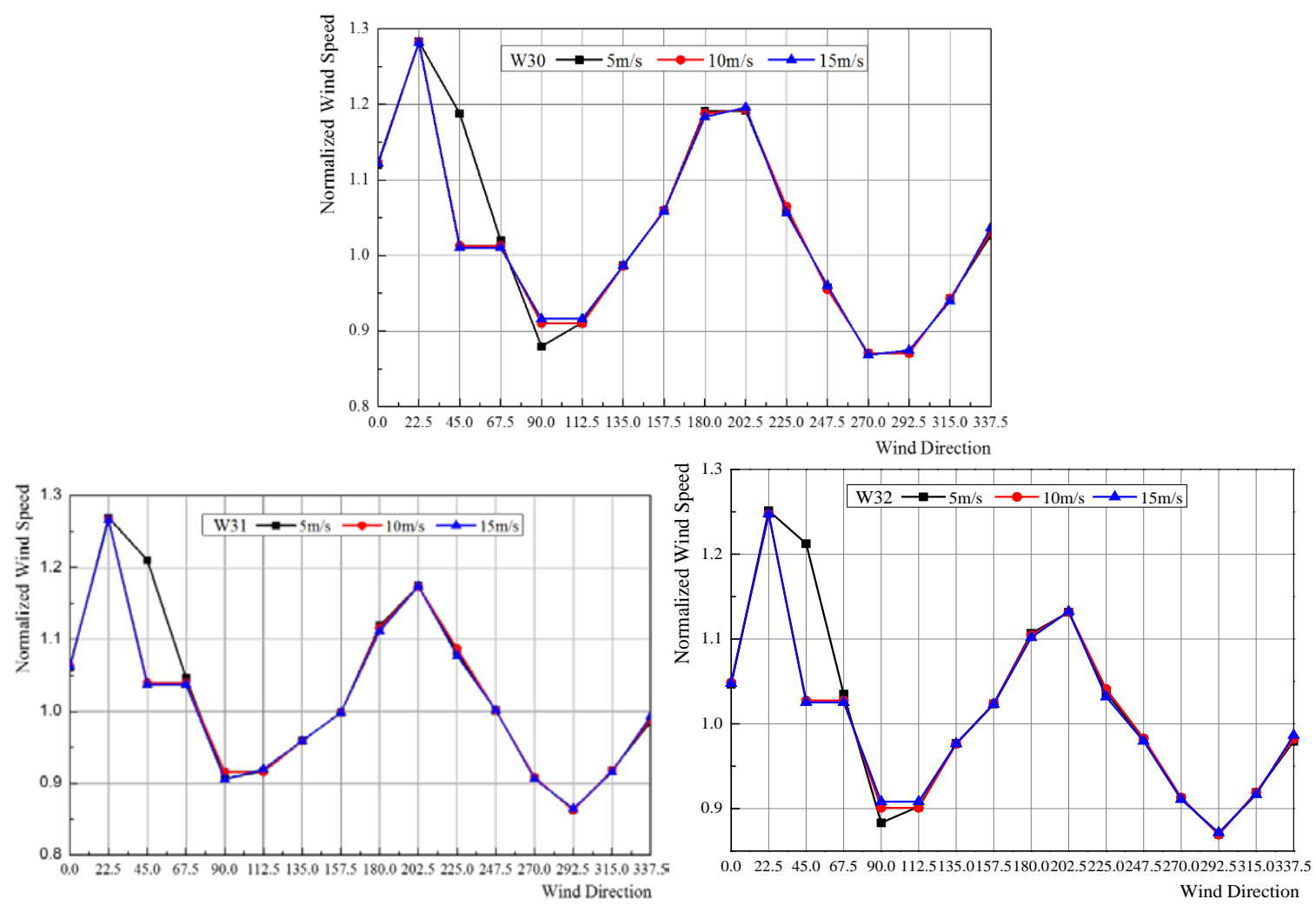

Fig.4 Contrast curves of the wind turbines under different incoming flow wind speed

\subsection{Measured Wind Speed Analysis}

Influence of wind direction towards acceleration effect. The contrast curves of observed normalized wind speed distribution in various sectors under the condition of incoming flow speed is $10 \mathrm{~m} / \mathrm{s}$ are shown in Fig.5. From Fig. 5 we can see a peculiar phenomenon that the wind speed is small even though that acceleration factor is big while the while the included angle is less than 45degree, especially while the included angle is less than 22.5 degree. The peculiar phenomenon indicates that 
the acceleration factor just depends on altitude, degree of terrain irregularity, roughness of ground surface and obstacles. However, the actual wind speed is also affected by atmospheric stability.

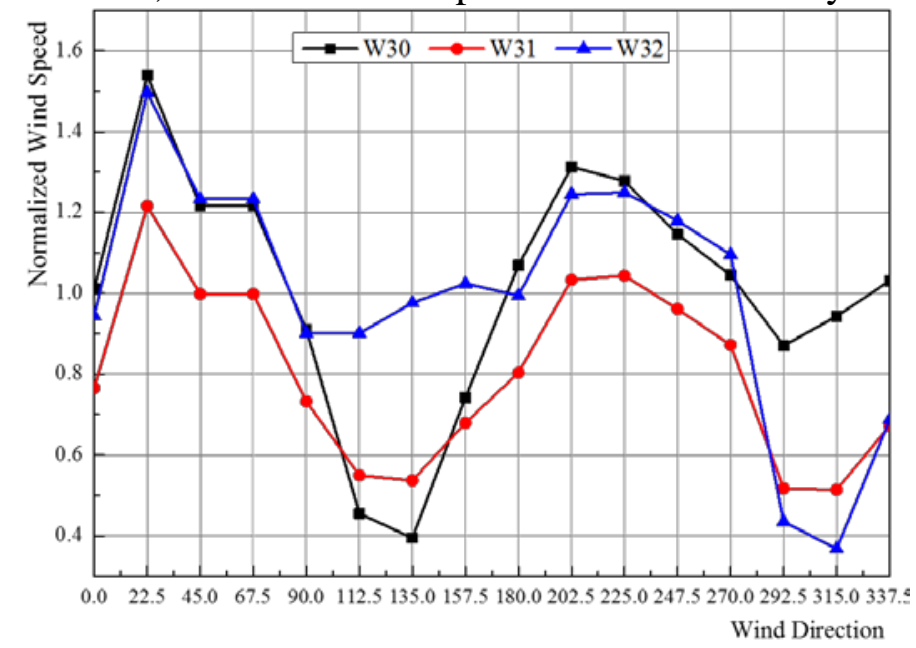

Fig.5 contrast curves of observed normalized wind speed distribution in various sectors

Influence of wind speed towards acceleration effect. Table 1 summarizes the statistical data of generated energy during 18 days while the wind direction in 3rd, 13th and 14th are 135 degree, the corresponding included angle is 0. Fig.6 shows the contrast curves of observed generated energy distribution during 18 days. Obviously, for different wind speed, the influence of speed towards acceleration effect is also different. There is nearly a positive correlation between them.

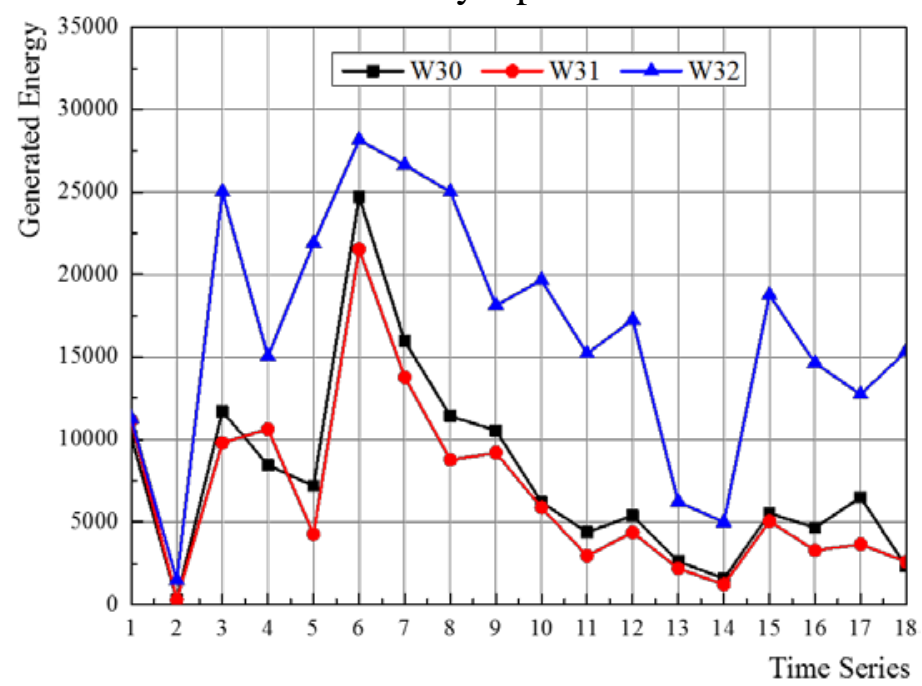

Fig.6 Contrast curves of observed generated energy distribution during 18 days

Table 1 Statistical data of generated energy (kW.h)

\begin{tabular}{cccccccc}
\hline Generated Energy & W30 & W31 & W32 & Generated Energy & W30 & W31 & W32 \\
\hline 1 & 10192 & 11176 & 11296 & 10 & 6217 & 5885 & 19666 \\
\hline 2 & 319 & 297 & 1483 & 11 & 4390 & 2969 & 15216 \\
\hline 3 & 11721 & 9818 & 25026 & 12 & 5400 & 4380 & 17271 \\
\hline 4 & 8466 & 10632 & 15050 & 13 & 2640 & 2195 & 6235 \\
\hline 5 & 7209 & 4279 & 21901 & 14 & 1579 & 1226 & 5002 \\
\hline 6 & 24714 & 21538 & 28163 & 15 & 5530 & 5060 & 18769 \\
\hline 7 & 15965 & 13786 & 26643 & 16 & 4674 & 3301 & 14652 \\
\hline 8 & 11435 & 8787 & 25015 & 17 & 6502 & 3662 & 12741 \\
\hline 9 & 10561 & 9213 & 18136 & 18 & 2316 & 2606 & 15365 \\
\hline
\end{tabular}




\subsection{Discussion}

From the above analysis, it is obvious that the simulation result carried out from turbulence model is not conform to the observed result very well, while the included angle is less than 45degree. The generated energy is overestimate by the CFD model. The acceleration effect at different wind turbine sites show a good concordance.

In the process of analyzing the wind distribution in a wind farm, the shunt phenomenon and backflow phenomenon are very common, while the included angle between ridge and wind direction is small relatively. But the CFD model cannot capture this phenomenon accurately. Therefore, the reliability of CFD simulation is relative low while in a complex terrain.

\section{Conclusions}

This work is a significative research to the practical engineering application of the micro-sitting of wind farms. While the intersection angle between the mountain ridge and wind direction is less than 22.5 degree, longitudinal spacing between wind turbines should be increased than the specifications and codes. In the process of wind farms micro-sitting, commercial software is not always reliable, and the experience of the engineers act complementary to it.

\section{References}

[1] Pep Moreno,Arne R Gravdahl,Manel Romero. Wind Flow over Complex Terrain:pplication of Linear and CFD Models [A].Madrid,pain, 003.

[2] Wood, N., 2000. Wind flow over complex terrain: a historical perspective and the prospect for large-eddy modelling.oundary-Layer Meteorol. 96, 11-32.

[3] Røkenes K, Krogstad P-A. Wind tunnel simulation of terrain effects on wind farm siting. Wind Energy 2009;12(4):391-410.

[4] Chagas G, Guedes RA, Manso MDO. Estimating wind resource using mesoscale modelling. In: European wind energy conference (EWEC); 2009.

[5] Carvalho D, Rocha A, Gomez-Gesteira M, Santos C. A sensitivity study of the WRF model in wind simulation for an area of high wind energy. Environ Modell Softw 2012;33:23-34.

[6] Stull RB. Transilient turbulence theory. Part I: The concept of eddy-mixing across finite distances. J AtmosSci 1984;41:3351-67.

[7] González J, Payán M, Santos J, González-Longatt F. A review and recent developments in the optimal wind-turbine micro-siting problem. Renew Sustain Energy Rev 2014;30:133-44.

[8] Bartl J. Wake measurement behind an array of two model wind turbines. M.S. Thesis, Royal Institute of Technology, Stockholm, Sweden; 2011. 